\title{
Molecular mechanisms of carbapenem resistance among Enterobacteriaceae isolated at Teaching Hospital, Batticaloa
}

\author{
MS Shihana ${ }^{1}$ V Francis ${ }^{2,3}$, LVC Liyanapathirana $^{4}$
}

\section{Introduction}

Family enterobacteriaceae include a group of Gram negative facultative anaerobes. Members of family enterobacteriaceae can develop resistance to $\beta$ - lactam antibiotics including carbapenems. The present study was carried out to identify the presence of selected genetic determinants of carbapenem resistance and extended spectrum beta lactamases (ESBLs) in carbapenem nonsusceptible enterobacteriaceae at Teaching Hospital, Batticaloa.

\section{Methods}

Isolates which were resistant or intermediate sensitive to any of the carbapenems using routine sensitivity testing were collected for a six-month period from $1^{\text {st }}$ October 2015 to $30^{\text {th }}$ March 2016, sub cultured on blood agar and MacConkey agar and, incubated at $37^{\circ} \mathrm{C}$ for $18-24$ hours in ambient air. Susceptibility testing was repeated using the disc diffusion method according to the CLSI guidelines. PCR amplification of bla $a_{\mathrm{TEM}}$, bla $a_{\mathrm{SHV}}$ and $b l a_{\mathrm{CTX}}-\mathrm{M}$ as potential genetic determinants for ESBLs and bla $a_{\mathrm{OXA}}-48$, bla $a_{\mathrm{KPC}}$ and bla $\mathrm{NDM}$ as genetic determinants for carbapenemases were performed.

\section{Results}

Total of 768 enterobacteriaceae were isolated during the study period and of these 5 were confirmed as resistant to the carbapenem (CRE) group. All the isolates were intermediate resistant or resistant to imipenem, meropenem, ertapenem, ceftazidime, cefotaxime, ciprofloxacin, levofloxacin and aztreonam. Four isolates carried at least one potential genetic determinant of ESBLs (bla $a_{\mathrm{CTX}-\mathrm{M}}$ in 3, bla $a_{\mathrm{TEM}}$ in 3 and bla $_{\mathrm{SHV}}$ in 2 isolates). Four isolates were found to carry at least one of the genetic determinants of carbapenemases tested for (bla $a_{\mathrm{OXA}}-48$ in 3 and bla $_{\mathrm{NDM}}$ in $1)$.

\section{Conclusions}

Metallo beta lactamase producing CRE are present in the Eastern region.

Keywords: carbapenemases, enterobacteriaceae, Carbapenem resistance

\footnotetext{
${ }^{1}$ Postgraduate Institute of Science, University of Peradeniya, Sri Lanka

${ }^{2}$ Teaching Hospital, Batticaloa, Sri Lanka

${ }^{3}$ Department of Pathophysiology, Faculty of Healthcare Sciences, Eastern University of Sri Lanka

${ }^{4}$ Department of Microbiology, Faculty of Medicine, University of Peradeniya, Sri Lanka

Address for correspondence: MS Shihana Telephone:+94761050160 Email: shihanams@yahoo.com 